\title{
EL UNIVERSO DE LA TEORIAA DE CONJUNTOS
}

IGNACTO JANÉ

Universidad Autónoma Metropolitana

IZTAPALAPA

Mi propósito en este artículo es presentar la axiomatización de la teoría de conjuntos llamada de Zermelo-Fraenkel, haciendo hincapié en su carácter intuitivo. A partir de una cierta intuición acerca de qué son y cómo se distribuyen los conjuntos, pretendo justificar cada uno de los axiomas. Una vez introducidos los axiomas mismos, nos adentraremos en algunas de sus consecuencias para recuperar -esta vez formalmente, expresado en teoremas de la teoría- el contenido intuitivo que nos sirvió de guía, contenido que se manifiesta especialmente en la estructura de la jerarquía acumulativa.

\section{La paradoja de Russell}

Todo conjunto es, ciertamente, una colección de objetos; en particular, de objetos que son conjuntos. Desde luego, esto no pretende ser una definición, ya que la noción de colección no es, ella misma, demasiado clara y, por otra parte, no estoy afirmando que conjuntos y colecciones coincidan. Como veremos, éste no es el caso. Sin embargo, algo preciso se puede concluir a partir de esta primera vaga aproximación a la naturaleza de los conjuntos. En primer lugar, que todo conjunto está determinado por sus elementos - pues ésta es una característica de las colecciones. La aserción de que todo conjunto está determinado por sus elementos es el principio de extensionalidad. Si $x$ es un objeto y si $a$ es un conjunto, escribamos ' $x \in a$ ' para indicar que $x$ es un elemento de $a$. Si $x$ no es un elemento de $a$ escribiremos ' $x \notin a$ '. Formalmente, el principio de extensionalidad se expresa como sigue: Si $a$ y $b$ son conjuntos,

$$
\forall x(x \in a \leftrightarrow x \in b) \rightarrow a=b .
$$

He tomado como punto de partida el lugar común de que todo conjunto es una colección. La paradoja de Russell puede emplearse aquí 
para mostrar que la afirmación opuesta, es decir, la afirmación de que toda colección es un conjunto; es falsa. Desde luego, la intuición de lo que sea una colección arbitraria no está bien delimitada, pero algo podemos decir acerca de colecciones, además del hecho de que están determinadas por sus elementos. Por ejemplo, toda propiedad de objetos determina una colección, a saber, su extensión. Pero el argumento de Russell muestra que hay propiedades cuya extensión no es un conjunto; de hecho, la propiedad de ser un conjunto que no se pertenece a sí mismo es una de ellas. El argumento, como es sabido, reza así: Sea $\mathrm{C}$ la colección de todos los conjuntos, $a$, tales que $a \notin a$. Así, para todo conjunto $a$,

$a \varepsilon \mathrm{C}$ si y sólo si $a \notin a$.

Si C fuera un conjunto, C sería un valor de la variable $a$, y, por tanto, podríamos concluir que

$$
C \varepsilon C \text { si y sólo si } C \notin C \text {, }
$$

lo cual es absurdo. Por tanto, $\mathrm{C}$ no es un conjunto.

\section{Estratos}

Si no toda colección de objetos es un conjunto, ¿qué colecciones lo son? Como ayuda a una respuesta más o menos satisfactoria, pensemos en cómo construimos conjuntos y observemos que, al formar un conjunto, disponemos ya de los objetos que serán sus miembros. En otras palabras, los posibles elementos del conjunto han sido construidos previamente.

Designemos con ' $V$ ' el universo de los conjuntos, o sea, la colección de todos los conjuntos, y limitemos nuestras consideraciones a conjuntos cuyos elementos son a su vez conjuntos —en breve, supongamos que todos los objetos son conjuntos. Un conjunto es, pues, a la vez un objeto y una colección. Una colección es un conjunto si y sólo si es un objeto, es decir, si y sólo și pertenece a $\mathrm{V}$, el universo.

El universo se estructura de un modo natural en estratos, de acuerdo con el orden de formación de los conjuntos. Usemos la letra ' $E$ ', con apóstrofos si es necesario, para referirnos a estratos. Un conjunto pertenece a un estrato dado si y sólo si sus elementos pertenecen a algún estrato anterior. Esto corresponde a la observación de que al construir un conjunto disponemos ya de sus elementos. Los estratos son, ellos 
mismos, conjuntos, y la relación de precedencia entre estratos es la rela. ción de pertenencia: $\mathbf{E}$ es anterior a $\mathbf{E}^{\prime}$ si y sólo si $\mathrm{E} \varepsilon \mathrm{E}^{\prime}$.

Todo conjunto se forma en algún estrato $y$, por tanto, pertenece a algún estrato. Es decir

$$
\forall x J E(x \in \mathrm{E}) \text {. }
$$

Nos referimos a (ii) como al principio de restricción.

Si $a$ es un conjunto, también lo serán todas sus subcolecciones; de hecho cualquier subcolección de $a$ puede construise al construir $a$, ya que sus elementos han sido formados previamente a la construcción de $a$. Puesto que toda propiedad, $\varphi$, determina una colección, estas consideraciones justifican el llamado principio de separación: $\mathrm{Si} a$ es un conjunto, entonces hay un conjunto, $b$, cuyos elementos son exactamente los elementos de $a$ con la propiedad $\varphi$. En símbolos,

$$
\forall a \operatorname{IB} b x(x \varepsilon b \leftrightarrow x \varepsilon a \wedge \varphi(x)) .
$$

Hemos visto que los conjuntos pertenecientes a un estrato, E, son colecciones de objetos pertenecientes a estratos anteriores. Por otra parte, E contendrá como elementos todas estas colecciones. Puesto que, si el objeto $a$ ha sido formado en un estrato anterior a $\mathrm{E}$, $a$ será formado nuevamente en $\mathrm{E}$, concluimos que todo estrato debe satisfacer el siguiente principio de acumulación:

$$
\forall x\left(x \in \mathrm{E} \leftrightarrow\left(\mathrm{HE}^{\prime} \varepsilon \mathrm{E}\right)\left(x \in \mathrm{E}^{\prime} \vee x \subseteq \mathrm{E}^{\prime}\right)\right)
$$

(Donde, en general, la notación ' $x \subseteq y$ ' es una abreviatura de la fórmula ' $\forall u(u \varepsilon x \rightarrow u \varepsilon y)$ '.)

\section{Axiomas de construcción}

Los axiomas de la teoría de Zermelo-Fraenkel se acostumbran clasificarlos en dos categorias: los de construcción y los de extensión. En términos de estratos, los axiomas de construcción incluirían los principios mencionados en el apartado anterior, ya que ellos describen, por lo menos en parte, cómo los conjuntos se distribuyen en estratos y cómo los estratos se relacionan entre sí. De hecho, los principios anteriores proveen más información de lo que pudiera pensarse tras un examen rutinario de su contenido, ya que implican que la colección de los estratos está ordenada, más aún, bien ordenada, por la relación de precedencia (o sea, de pertenencia) entre estratos. 
Sin embargo, las axiomatizaciones comunes de la teoría de ZermeloFraenkel no mencionan estratos en absoluto. Una presentación usual de los axiomas de construcción es la siguiente:

Axioma de extensionalidad:

$$
\forall a \forall b(\forall x(x \in a \leftrightarrow x \varepsilon b) \rightarrow a=b)
$$

Esquema de separación:

$$
\forall a \operatorname{G} b \forall x(x \in b \leftrightarrow x \varepsilon a \wedge \varphi(x))
$$

Axioma del par:

$$
\forall a \forall b \exists c \forall x(x \& c \leftrightarrow x=a \vee x=\dot{b})
$$

Axioma de la unión:

$$
\forall a \exists b \forall x(x \in b \leftrightarrow \exists z(x \in z \wedge x \quad b \quad \varepsilon a))
$$

Axioma del conjunto potencia:

$$
\forall a \mathrm{H} b \forall x(x \in b \leftrightarrow x \subseteq a)
$$

Esquema de regularidad:

$$
\exists x \varphi(x) \rightarrow \exists x(\varphi(x) \wedge 7 \exists y(y \varepsilon x \wedge \varphi(y)))
$$

Observemos que estos axiomas son en número infinito, ya que hay un axioma de separación y un axiomá de regularidad para cada propiedad especificable -0 , más precisamente, para cada fórmula, $\varphi$, del lenguaje de teoría de conjuntos (el lenguaje de primer orden con identidad cuyo único símbolo no lógico es el relator binario ' $\varepsilon$ ').

Los axiomas de extensionalidad y de separación han sido ya discutidos anteriormente. Con respecto al de separación, dada la fórmula $\varphi$ y dado un conjunto $a$, denotemos por ' $\{x \varepsilon a: \varphi(x)\}$ ' el conjunto, $b$, cuya existencia tal axioma garantiza. El axioma del par afirma la existencia, para cualesquiera conjuntos $a, b$, de su par, $\{a, b\}-e l$ conjunto cuyos únicos elementos son $a$ y $b$. El axioma de la unión garantiza, para todo conjunto, $a$, la existencia de $\mathbf{U} a$, su unión, a saber, del conjunto cuyos elementos son los elementos de los elementos de $a$. (En particular, pues, si $a$ y $b$ son conjuntos, el conjunto $a \cup b$-cuyos elementos son los 
elementos de $a$ y los elementos de $b$ - existe, ya que $a \cup b=\mathbf{U}\{a, b\}$ ). $\mathrm{El}$ axioma del conjunto potencial afirma que, dado un conjunto $a$, su conjunto potencia, $\mathrm{P} a$, también existe — donde $\mathrm{P} a$ es el conjunto cuyos elementos son precisamente los subconjuntos de $a$. (El nombre de "conjunto potencia' hace referencia al hecho de que, si $a$ es finito con $n$ elementos, entonces $\mathrm{Pa}$ tiene $2^{n}$ elementos). Finalmente (en orden, que no en importancia) está el axioma de regularidad. Dice simplemente que si algún conjunto posee la propiedad $\varphi$, entonces hay un conjunto minimal - con respecto a la relación de pertenencia- que posee tal propiedad.

¿Por qué debemos aceptar estos axiomas de construcción como verdaderos de nuestra idea intuitiva de conjunto? Desde luego, se podría argumentar, independientemente de lo dicho en las secciones anteriores, en favor de su validez. Pero no es necesario. Tales axiomas se deducen de los cuatro principios fundamentales - dos sobre conjuntos en general, dos sobre estratos en particular - presentados en las secciones anteriores. Por tanto, es innecesario indagar más profundamente en nuestra intuición de los conjuntos para justificar tales axiomas.

Al iniciar esta sección mencioné que los principios (i)-(iv) implican que los estratos están bien ordenados por la relación de pertenencia, o sea, que las siguientes aserciones son consecuencia de tales principios:

$$
\begin{aligned}
& \forall E(E \notin E) \\
& \forall E V E^{\prime} \forall E^{\prime \prime}\left(E \varepsilon E^{\prime} \wedge E^{\prime} \varepsilon E^{\prime \prime} \rightarrow E \varepsilon E^{\prime \prime}\right) \\
& \forall E V E^{\prime}\left(E \varepsilon E^{\prime} \vee E^{\prime} \varepsilon E \vee E=E^{\prime}\right) \\
& \operatorname{JE} \psi(E) \rightarrow \operatorname{GE}\left(\psi(E) \wedge\left(7 E^{\prime}\right)\left(E^{\prime} \varepsilon E \wedge \psi\left(E^{\prime}\right)\right)\right)
\end{aligned}
$$

Aquí voy a probar sólo (1). (El resto de la prueba puede encontrarse en [ScotT].) Supongamos que (I) es falso. Sea, pues, E un estrato tal que $\mathrm{E} \varepsilon \mathrm{E}$. Consideremos el conjunto

$$
a=\{x \in \mathrm{E}: x \notin x\}
$$

(a ciertamente existe, por separación). Por definición de $a, a \subseteq \mathrm{E}, \mathrm{y}$, ya que suponemos que $\mathbf{E} \varepsilon \mathrm{E}$, concluimos, haciendo uso del principio de acumulación, que $a \varepsilon \mathrm{E}$. Pero entonces $a \varepsilon a$ si y sólo si $a \notin a$. Esto es absurdo. Por tanto, $\mathrm{E} \& \mathrm{E}$.

La deducción de los axiomas de construcción de la teoría de ZermeloFraenkel a partir de (i).(iv) es ahora relativamente sencilla. El axioma 
del par se prueba así: Sean $a, b$ conjuntos. Por restricción, hay estratos $\mathrm{E}, \mathrm{E}^{\prime}$, tales que $a \varepsilon \mathrm{E}, b \varepsilon \mathrm{E}^{\prime}$. Por (3). podemos suponer que $\mathrm{E} \varepsilon \mathrm{E}^{\prime}$ o $\mathbf{E}=\mathrm{E}^{\prime}$. Por tanto, por acumulación, $a, b \in \mathrm{E}^{\prime}$. Pero entonces, $\{a, b\}=$ $\left\{x \in E^{\prime}: x=a \vee x=b\right\}$ existe por separación.

Para justificar el axioma de la unión razonemos como sigue. Veamos primeramente que, para todo conjunto $x, y$ para todo estrato $\mathrm{E}$,

$$
x \varepsilon \mathrm{E} \rightarrow x \subseteq \mathrm{E} .
$$

Pues supongamos que $x \in \mathrm{E}$. Por (4) hay un $\mathrm{E}^{\prime}$ tal que $x \in \mathrm{E}^{\prime}$ y para todo estrato $\mathrm{E}^{\prime \prime} \varepsilon \mathbf{E}^{\prime}, x \notin \mathrm{E}^{\prime \prime}$. Por (3), $\mathrm{E}^{\prime} \varepsilon \mathrm{E} \circ \mathrm{E}^{\prime}=\mathrm{E}$, de donde, por acumulación, $\mathrm{E}^{\prime} \subseteq \mathrm{E}$. Puesto que $x \varepsilon \mathrm{E}^{\prime}$, hay un $\mathrm{E}^{\prime \prime} \varepsilon \mathrm{E}^{\prime}$ tal que $x \subseteq \mathrm{E}^{\prime \prime}$ o $x \varepsilon \mathrm{E}^{\prime \prime}$. Pero $x \varepsilon \mathrm{E}^{\prime \prime}$ es imposible, por la minimalidad de $\mathrm{E}^{\prime}$. Por tanto, $x \subseteq \mathrm{E}^{\prime \prime}$. Por (3) y por acumulación, $\mathrm{E}^{\prime \prime} \subseteq \mathrm{E}^{\prime} \subseteq \mathrm{E}$. De donde $x \subseteq \mathrm{E}$.

Probemos ahora el axioma de la unión. Sea $a$ un conjunto. Por restricción hay un estrato, $\mathrm{E}$, tal que $a \varepsilon \mathrm{E}$. Por (5), $a \subseteq \mathrm{E}$, de modo que si $x \in a$, entonces $x \in \mathrm{E}$, y, de nuevo por (5), $x \subseteq \mathrm{E}$. Por tanto, si $z \varepsilon x$ y $x \in a$, entonces $z \in \mathrm{E}$. Por consiguiente,

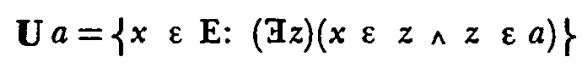

existe por separación.

El axioma del conjunto potencia se justifica como sigue: Sea $a$ un conjunto. Sea $\mathrm{E}$ un estrato tal que $a \in \mathrm{E}$. Usando (4) como en la prueba de (5) vemos que hay un $\mathrm{E}^{\prime} \varepsilon \mathrm{E}$ tal que $a \subseteq \mathrm{E}^{\prime}$. Por tanto, si $z \subseteq a$, entonces $z \subseteq \mathrm{E}^{\prime}$ y $z \in \mathrm{E}$. Pero entonces

$$
\mathrm{P} a=\{z \varepsilon \mathbf{E}: z \subseteq a\}
$$

existe por separación.

Justifiquemos, finalmente, el axioma de regularidad. Supongamos que $\exists x \varphi(x)$ y pongamos

$$
\psi(\mathbf{E}) \underset{\mathrm{def}}{\leftrightarrow} \exists x(x \in \mathrm{E} \wedge \varphi(x))
$$

Por restricción, dado que $\mathbf{I} x \varphi(x)$, tenemos que $\mathbf{I E} \psi(\mathrm{E})$. Por tanto, por (4), hay un E minimal con la propiedad $\psi$-es decir, un $E$ tal que

$$
\psi(E) \quad y \quad\left(7 \exists E^{\prime}\right)\left(E^{\prime} \varepsilon E \wedge \psi\left(E^{\prime}\right)\right) .
$$

Fijemos tal E. Hay, pues, un conjunto, $x$, tal que

$$
\begin{array}{llll}
x \in E & \mathrm{y} & \varphi(x) .
\end{array}
$$


Sea $y$ un elemento cualquiera de $x$. Queremos comprobar que $7 \varphi(y)$. Por acumulación, hay un $\mathbf{E}^{\prime} \varepsilon \mathbf{E}$ tal que $y \in \mathbf{E}^{\prime}$. Pero entonces $7 \varphi(y)$, ya que, en otro caso, $\psi\left(E^{\prime}\right)$-en contradicción con la minimalidad de $E$.

\section{Los axiomas de extensión}

Hemos visto que nuestros cuatro principios fundamentales son suficientes para la justificación de los axiomas de construcción de la teoría de Zermelo-Fraenkel. No es difícil ver que tales principios nos garantizan la existencia de todos los conjuntos hereditariamente finitos (o sea, con bastante imprecisión, de los conjuntos finitos cuyos elementos son finitos, los elementos de cuyos elementos son finitos, etc.). Por otra parte, esto es todo lo que nos garantizan, pues si definimos recursivamente sobre los números naturales intuitivos:

$$
\begin{aligned}
& E_{0}=\phi \\
& E_{n+1}=P\left(E_{n}\right)
\end{aligned}
$$

(donde $\phi$ es el conjunto vacío - -el cual existe, por separación), entonces la colección de todos los conjuntos que pertenecen a algún $\mathbf{E}_{\mathbf{n}}$ (entre los que se cuentan los $E_{n}$ mismos, ya que $E_{n} \varepsilon E_{n+1}$ ) constituye un modelo de nuestros cuatro principios fundamentales y, por tanto, de los axiomas de construcción de la teoría de Zermelo-Fraenkel.

Así, estos axiomas son insuficientes para la fundamentación de la matemática, ya que esta disciplina necesita conjuntos infinitos en profusión. Hace falta, pues, agregar axiomas que aseguren, por lo menos, la existencia de algún conjunto infinito.

Notemos que, en el modelo anterior, si bien cada estrato (cada $E_{n}$ ) es finito, hay sin embargo un número infinito de estratos y, por tanto, un número infinito de conjuntos. Si hubiera, pues, un estrato, $\mathrm{E}$, posterior a todos los $\mathrm{E}_{\mathrm{n}}$ (es decir, un estrato, $\mathrm{E}$, tal que, para todo $n, \mathrm{E}_{\mathbf{n}} \varepsilon \mathrm{E}$ ), tal estrato sería infinito ya que, por acumulación, todo conjunto perteneciente a algún $E_{n}$ pertenecería también a $E$.

¿Cómo formular un axioma de infinitud? Es decir, ¿cómo afirmar la existencia de un conjunto infinito? Observemos que si hubiera un estrato menor, $\mathrm{E}$, posterior a todos los $\mathrm{E}_{\mathrm{n}}$, entonces $\phi \varepsilon \mathrm{E}$ (de hecho, $\phi \varepsilon \mathrm{E}_{1}$ ) $\mathrm{y}$, para todo $x \in \mathrm{E}, x \cup\{x\} \varepsilon E$ (pues si $x \in \mathrm{E}_{\mathrm{n}}$, entonces, por (5), $x \cup\{x\} \subseteq \mathrm{E}_{\mathrm{n}} \mathrm{y}$, por tanto, $\left.x \cup\{x\} \varepsilon \mathrm{P}\left(\mathrm{E}_{\mathrm{n}}\right)=\mathrm{E}_{\mathrm{n}+1}\right)$. Por otra parte, si $x \neq y$ entonces, por regularidad, $x \cup\{x\} \neq y \cup\{y\}$; de modo que no sólo $\mathrm{E}$, sino cualquier conjunto que contenga como elemento al conjunto vacío, y tal que siempre que contenga a un conjunto $x$ contiene tam- 
bién a $x \cup\{x\}$, será infinito. El axioma de infinitud, en su forma usual, es simplemente la afirmación de la existencia de un tal objeto.

Axioma de infinitud:

$$
\exists a(\phi \varepsilon a \wedge \forall x(x \in a \rightarrow x \cup\{x\} \varepsilon a)) .
$$

La teoría descrita hasta ahora - la teoría cuyo único principio de extensión es el axioma de infinitud - es la llamada teoría de Zermelo. Esta teoria es suficiente para el desarrollo de prácticamente toda la matemática tradicional, en especial del análisis en los espacios euclidianos.

Sin embargo, desde un punto de vista intuitivo, la teoría de Zermelo deja algo que desear: no garantiza la existencia de suficientes estratos. Seré más preciso a este respecto.

Hemos visto que el axioma de infinitud nos proporciona estratos infinitos. Puesto que los estratos están bien ordenados, hay un primer estrato infinito (éste será el primer estrato que satisface el a ioma de infinitud, según vimos). Llamemos ' $\mathrm{E}$ "' a tal estrato, el cual no es más que la unión de los $\mathbf{E}_{\mathrm{n}}$. Nuevamente por recursión sobre los números naturales intuitivos definamos:

$$
\begin{aligned}
& \mathbf{E}_{0}^{\#}=\mathbf{E}^{\#} \\
& \mathbf{E}_{n+1}^{\#}=\mathbf{P}\left(\mathbf{E}_{n}^{\#}\right) ;
\end{aligned}
$$

entonces, la colección de todos los conjuntos que pertenecen a algún $E_{n}^{\#}$ (entre los que se contarán, en particular, todos los $E_{n} y$ todos los $E_{n}^{\#}$ ) es un modelo de los axiomas de Zermelo. Sin embargo, desde un punto de vista intuitivo, hay muchas colecciones que deberian ser conjuntos y que no pertenecen a ningún $E_{n}^{\#}$. Algunas de ellas (por ejemplo, el ordinal $\omega+\omega)$ son tan fácilmente definibles que claman por una extensión del sistema. Sea $\omega$ el menor (con respecto a la inclusión) conjunto que satisface el axioma de infinitud. $\omega$ no es más que el correlato formal, en la teoría, del conjunto de los números naturales, ya que si identificamos los números naturales intuitivos con conjuntos, de modo que

$$
\begin{aligned}
& \mathbf{0}=\boldsymbol{\phi} \\
& \mathbf{n}+\mathbf{1}=\mathbf{n} \cup\{\mathbf{n}\},
\end{aligned}
$$




$$
\omega=\{0,1,2, \ldots\}
$$

y $\omega \varepsilon \mathrm{E}_{1}^{\#}$.

Si continuamos este proceso intuitivo definiendo

$$
\begin{aligned}
& \omega+1=\omega \cup\{\omega\} \\
& \omega+\mathrm{n}+1=\omega+\mathrm{n} \cup\{\omega+\mathrm{n}\}
\end{aligned}
$$

entonces

$$
\omega+\omega=\omega \cup\{\omega, \omega+1, \omega+2, \omega+3, \ldots\}
$$

$\omega+\omega$ es manifiestamente una colección rãzonablemente simple que, sin embargo, no pertenece a ningún $\mathrm{E}_{\mathrm{n}}^{\#}$ y cuya existencia, por tanto, no puede probarse a partir de los axiomas de Zermelo. Notemos que cada elemento de $\omega+\omega$ sí pertenece a algún $\mathrm{E}_{n}^{\#}$-de hecho, $\omega+\mathrm{n} \varepsilon \mathrm{E}_{n+1}^{\#}$. El problema con $\omega+\omega$ es que para la construcción de todos sus elementos se requieren todos los estratos $\mathrm{E}_{\mathrm{n}}^{\#}$, de modo que no hay ningún 'estrato en el que $\omega+\omega$ pueda realizarse como conjunto. Para asegurar la existencia de $\omega+\omega$ en la teoría bastaría postular (al igual que en el caso del axioma de infinitud) la existencia de un nuevo estrato, diga-

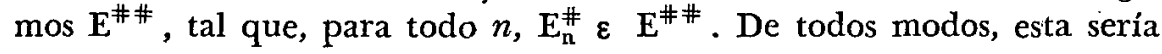
una solución ineficaz: no es difícil ver que podríamos parafrasear el argumento anterior para mostrar que hay todavía colecciones que intuitivamente debieran ser conjuntos y cuya existencia no sería probable. (El nuevo ejemplo sería, en este caso, el ordinal $\omega+\omega+\omega$.)

Lo que se requiere, pues, es un axioma no local, sino global, de infinitud que nos garantice que la cadena ascendente de estratos no se detendrá inoportunamente. El problema con los $\mathrm{E}_{n}^{\#}$ residía en que cada uno de ellos estaba en correspondencia con un número natural (su subindice) y que, si bien la colección de los números naturales (o su correlato formal en la teoría) constituye un conjunto, la colección de los $E_{n}^{\#}$ no lo constituye necesariamente. Esta consideración sugiere un procedimiento para solucionar no sólo este problema concreto sino todos los problemas afines. El principio global de infinitud que necesitamos es una propuesta del siguiente tipo: Sea $a$ un conjunto y supongamos que a cada $x \in a$ hemos asociado un estrato $\mathrm{E}_{\mathbf{x}}$. Entonces la colección de los $E_{x}$ es también un conjunto, o, equivalentemente, hay un estrato posterior a todos los estratos $\mathbf{E}_{\mathbf{x}}$.

Formulada en términos de conjuntos _ sìn mencionar estratos-, la 
propuesta anterior es el llamado axioma de substitución y se debe a Fraenkel y a Skolem. Para su formulación debemos precisar la frase "a cada elemento $x \in a$ hemos asignado un (estrato $E_{x}$ ) conjunto $y_{x}$ ". Lo que esto significa es que tenemos una "relación funcional" que hace corresponder un elemento $y_{x}$ a cada $x \in a$. Tal "relación funcional" puede identificarse con una fórmula de dos variables libres $\varphi(x, y)$ tal que para todo $x$ haya un solo $y$ que satisfaga $\varphi(x, y)$. Puesto que para cada fórmula de este tipo tendremos una relación funcional, y puesto que en la teoria no podemos cuantificar sobre fórmulas, hay que formular el principio de substitución como esquema.

Esquema de substitución:

$$
\begin{aligned}
& \forall x \forall y \forall z(\varphi(x, y) \wedge \varphi(x, z) \rightarrow y=z) \rightarrow \\
& \forall a \exists b \forall y(y \varepsilon b \leftrightarrow \mathbb{A} x(x \varepsilon a \wedge \varphi(x, y))) .
\end{aligned}
$$

El conjunto, $b$, cuya existencia garantiza el esquema de substitución, suele denotarse por

$$
\{y:(\exists x \varepsilon a) \varphi(x, y)\} .
$$

Con esto concluyo la presentación formal y la justificación intuitiva de los axiomas de Zermelo-Fraenkel. Quisiera observar, sin embargo, que 19) el esquema de separación es un corolario del de substitución; 29) el esquema de regularidad puede formularse como axioma, y 39) la teoría de Zermelo-Fraenkel no es finitamente axiomatizable - de modo que, por $1^{9}$ ) y $\left.2^{\circ}\right)$, el esquema de substitución no es reemplazable por un axioma.

\section{La jerarquia cumulativa}

La justificación intuitiva aquí presentada de la teoria de ZermeloFraenkel se basó en el concepto de estrato. Pero en la formulación misma de los axiomas no se hace mención alguna de tal concepto. Mi intención ahora es mostrar que, sin embargo, el concepto de estrato es recuperable en la teoria de tal modo que las propiedades básicas que intuitivamente asignamos a los estratos y a su interrelación sean probables en la teoría.

Éste es, a mi parecer, un punto importante para la comprensión de la teoría de conjuntos. La definibilidad de la jerarquia cumulativa y el hecho, probable en Zermelo-Fraenkel, de que todo conjunto pertenece a algún estrato de esta jerarquía, hace difícil mantener la opinión, tan comúnmente expresada, de que la axiomatización de Zermelo-Fraenkel 
es ad hoc. Según esta opinión, nuestros axiomas han sido elegidos simplemente con dos fines en mente: 19) evitar la reproducción de los argumentos que conducen a las paradojas y $2^{\circ}$ ) proporcionar a los matemáticos instrumentos de construcción y prueba suficientes para sus necesidades cotidianas. Si estas fueran las únicas directrices de la axiomatización aqui presentada, sería difícil explicar cómo surge, en la teoría misma, esta distribución jerárquica de los conjuntos en estratos de acuerdo con su complejidad.

Para introducir formalmente la jerarquía cumulativa debemos ocuparnos previamente de los ordinales y de los principios de inducción y recursión transfinita. No probaré nada acerca de estos conceptos. Me limitaré a mencionar y comentar las nociones que precise para la discusión de la jerarquía. Las pruebas no ofrecidas pueden encontrarse, por ejemplo, en [LEVY].

Un conjunto transitivo es, por definición, un conjunto, $a$, tal que los elementos de los elementos de $a$ son, a su vez, elementos de $a$-en otras palabras, tal que los elementos de $a$ son también subconjuntos de $a$. Un ordinal se define como un conjunto transitivo cuyos elementos son conjuntos transitivos. Así, por ejemplo, $\left.1^{\circ}\right) \phi$ es un ordinal, $\left.2^{\circ}\right)$ si $x$ es un ordinal también lo es $x \cup\{x\}$. (el llamado sucesor de $x$ ) y 39 ) si $A$ es un conjunto de ordinales, entonces $\mathbf{U} A$, la unión de $A$, es tambièn un ordinal.

Todo ordinal está ordenado, de hecho bien ordenado, por la relación, $\varepsilon$, de pertenencia. Usemos las primeras letras minúsculas del alfabeto griego para referirnos a ordinales. Así, definamos para ordinales

$$
\begin{aligned}
& \alpha<\beta \underset{\text { def }}{\leftrightarrow} \alpha \varepsilon \beta \\
& \alpha \leq \beta \overleftrightarrow{\text { def }} \alpha \varepsilon \beta \vee \alpha=\beta
\end{aligned}
$$

Todo ordinal es el conjunto de los ordinales menores que él. Hay un ordinal menor, a saber $\phi$. Para mayor conspicuidad definamos

$$
\begin{aligned}
& 0=\phi \\
& \alpha+1=\alpha \cup\{\alpha\}
\end{aligned}
$$

Entonces los números naturales no son más que los primeros ordinales, a saber: $0,1=0+1,2=1+1,3=2+1$, etc. Básicamente, los ordinales son una extensión de los números naturales en el dominio de lo transfinito. $\omega$, el conjunto de los números naturales, es el primer ordinal infinito. 
La colección de los ordinales está ordenada, y bien ordenada, por la relación de pertenencia. De hecho, la totalidad de los ordinales no es un conjunto, según la teoría de Zermelo-Fraenkel, por lo que la afirmación anterior necesita trabajarse. Precisamente, lo que quiero decir es que las aserciones siguientes son teoremas:

$$
\begin{aligned}
& \forall \alpha(\alpha \nless \alpha) \\
& \forall \alpha \beta \gamma(\alpha<\beta \wedge \beta<\gamma \rightarrow \alpha<\gamma) \\
& \forall \alpha \beta(\alpha<\beta \vee \beta<\alpha \vee \beta=\alpha) \\
& \exists \alpha \varphi(\alpha) \rightarrow \exists \alpha(\varphi(\alpha) \wedge(\urcorner \exists \beta)(\beta<\alpha \wedge \varphi(\beta)))
\end{aligned}
$$

(donde, en (9), $\varphi$ es cualquier fórmula del lenguaje de la teoría).

Observemos que, dado que $\varepsilon$ no es más que la relación de pertenencia entre ordinales, (9) es simplemente un caso particular del axioma de regularịdad. (9) dice que si algún ordinal posee la propiedad $\varphi$, entonces hay un ordinal menor que la posee. De hecho esta afirmación es la contrapositiva del principio de inducción para ordinales, formulable como sigue:

$$
\forall \alpha((\forall \beta<\alpha) \varphi(\beta) \rightarrow \varphi(\alpha)) \rightarrow \forall \alpha \varphi(\alpha)
$$

O sea, para mostrar que todo ordinal posee la propiedad $\varphi$, basta mostrar que un ordinal cualquiera, $\alpha$, posee tal propiedad - bajo el supuesto (la hipótesis inductiva) de que todo ordinal menor que $\alpha$ la posee.

El principio de inducción puede considerarse, pues, como un método de prueba. Pero, al igual que en el caso de los números naturales, la existencia de un buen orden permite la definición recursiva de operaciones en ordinales. Algo imprecisamente, para definir una operación, $O$, por recursión sobre los ordinales, basta mostrar cómo definir el valor, $O(\alpha)$, de tal operación para el ordinal $\alpha$ a partir de los valores $O(\beta)$ para cada $\beta<\alpha$. La definición de $O(\alpha)$ a partir de $\operatorname{los} O(\beta)(\beta<\alpha)$ debe ser uniforme -o sea, debe ser la misma en cada caso, independientemente de $\alpha$. Reconozco que esta explicación es vaga. La he ofrecido para dar un aire de naturalidad a la discusión más formal y precisa que ahora presento.

Supongamos que disponemos de una operación, $G$, que asigna a cada conjunto, $a$, un conjunto $\mathrm{G}(a)$. (Tal operación se dará en general mediante una fórmula del lenguaje conjuntista, de modo semejante a lo que ocurre en la formulación de los axiomas de substitución. De hecho, 
una operación en este contexto no es más que lo que alli llamé una "relación funcional"). Una forma del teorema (o, mejor, esquema) de definición por recursión sobre ordinales afirma entonces la definibilidad de una única operación, $O$, tal que, para todo ordinal, $\alpha$,

$$
O(\alpha)=G(\{, O(\beta): \beta<\alpha\})
$$

Asi, $O(\alpha)$ se obtiene aplicando la "regla" G (siempre la misma) al conjunto de los valores $O(\beta)$ para $\beta<\alpha$.

Quisiera observar que ésta no es la forma más general del esquema de recursión sobre ordinales, pero es suficiente para mis propósitos presentes.

Con el teorema de recursión en nuestro haber, estamos ya en disposición de introducir formalmente la jerarquía cumulativa. Definamos por recursión sobre los ordinales:

$$
V(\alpha)=\mathbf{U}\{P(V(\beta)): \beta<\alpha\}
$$

Antes de seguir adelante, veamos que (11) es un caso particular de (10), de modo que nuestra definición está justificada. Desde luego, ' $V$ ' juega aquí el papel de ' $O$ '. Definamos la operación $G$ mediante la ecuación:

$$
G(x)=\mathbf{U}\{P(y): y \quad \varepsilon x\}
$$

(o, si queremos ser más formales, sea $\varphi(x, z)$ la fórmula siguiente:

$$
\forall u(u \varepsilon z \leftrightarrow(\Pi y)(y \varepsilon x \wedge u \subseteq y))
$$

no es difícil ver que para todo conjunto $x$ hay exactamente un conjunto $\underline{z}$ tal que $\varphi(x, z)$ y que

$$
\mathrm{G}(x)=z \text { si y sólo si } \varphi(x, z)) \text {. }
$$

Entonces, ya que $\beta \varepsilon \alpha$ si y sólo si $\beta<\alpha$,

$$
V(\alpha)=G(\{V(\beta): \beta<\alpha\}) ;
$$

o sea, que (11) es un caso particular de (10).

Pasemos a analizar los conjuntos $V(\dot{\alpha})$ definidos según(11). Escribamos ' $V \alpha$ ' en vez de ' $V(\alpha)$ '. 
En primer lugar, observemos que (11) se puede reformular comó

$$
\forall x\left(x \varepsilon V_{\alpha} \leftrightarrow(\exists \beta<\alpha)\left(x \subseteq V_{\beta}\right)\right) \text {. }
$$

Supongamos que $\beta<\alpha$. Como $V_{\beta} \subseteq V_{\beta}$, (11') nos permite concluir que $V_{\beta} \varepsilon V_{\alpha}$. Por otra parte, si $x \in V_{\beta}$, entonces $x \subseteq V_{\gamma}$, para algún $\gamma<\beta$. Por tanto, $x \subseteq V_{\gamma}$, para algún $\gamma<\alpha$. De donde $x \varepsilon \mathrm{V}_{\alpha}$. Hemos probado, pues,

$$
\beta<\alpha \rightarrow V_{\beta} \varepsilon V_{\alpha}
$$

y

$$
\beta<\alpha \rightarrow V_{\beta} \subseteq V_{\alpha}
$$

De $\left(12^{\prime}\right)$ se sigue que

$$
\forall x\left(x \in V_{\alpha} \rightarrow x \subseteq V_{\alpha}\right)
$$

pues sea $x \in \mathrm{V}_{\alpha}$. Hay, pues, $\beta<\alpha$ tal que $x \subseteq \mathrm{V}_{\beta}$. Pero $\mathrm{V}_{\beta} \subseteq \mathrm{V}_{\alpha}$; por tanto $x \subseteq \mathrm{V}_{\alpha}$.

- Con la ayuda de (13) y (12), (11') es equivalente a

$$
\forall x\left(x \in V_{\alpha} \leftrightarrow\left(\nexists V_{\beta} \varepsilon V_{\alpha}\right)\left(x \in V_{\beta} \vee x \subseteq V_{\beta}\right)\right)
$$

lo cual no es más que nuestro viejo principio de acumulación formulado en términos de los $V_{\alpha}$. Así, para poder afirmar justificadamente que los $V_{\alpha}$ son el correlato formal de los estratos, debemos comprobar solamente que satisfacen el principio de restricción:

$$
\forall x \quad \mathcal{I} \alpha\left(x \in V_{\alpha}\right) \text {. }
$$

Sea $x$ un conjunto cualquiera. Procediendo por contradicción, supongamos que $7 \exists \alpha\left(x \in V_{\alpha}\right)$. Por el axioma de regularidad hay un conjunto minimal, $x_{0}$, tal que $7 \exists_{\alpha}\left(x_{0} \varepsilon V \alpha\right)$. Es decir, hay un conjunto $x_{0}$ tal que

$$
\text { ᄀษ } \alpha\left(x_{0} \varepsilon V_{\alpha}\right)
$$

pero

(\#\#)

$$
\forall y\left(y \varepsilon x_{0} \rightarrow \exists \beta\left(y \varepsilon V_{\beta}\right)\right) .
$$

Puesto que los ordinales están bien ordenados (satisfacen (9) en par- 
ticular), para cada $y \varepsilon x_{0}$ (por (\#:\#)), hay un ordinal menor, $\beta(y)$, tal que $y \& V_{\beta(y)}$.

Por el axioma de sustitución, el conjunto

$$
\mathrm{A}=\left\{\beta(y): y \varepsilon x_{0}\right\}
$$

existe. Ahora bien, $\mathrm{A}$ es un conjunto de ordinales. Por tanto, según lo indicado después de la definición de ordinal, $\mathbf{U}$ A es también un ordinal; digamos $\alpha_{0}=\mathbf{U}$ A. Además, para cada $y \varepsilon x_{0}, \beta(y) \leqslant \alpha_{0}$. Por tanto, por (12'), para cada $y \varepsilon x_{0}, V_{\beta(y)} \subseteq V_{\alpha_{0}}$. Pero, por definición de $\beta(y)$, $y \varepsilon \mathrm{V}_{\beta(y)}$. Así podemos concluir que, para todo $y \varepsilon x_{0}, y \varepsilon V \alpha_{0}$; o sea, que

$$
x_{0} \subseteq \mathrm{V} \alpha_{0} \text {. }
$$

Pongamos

$$
\alpha=\alpha_{0}+1 \text {. }
$$

Dado que $\alpha=\alpha_{0} \cup\left\{\alpha_{0}\right\}$, es obvio que

$$
\boldsymbol{\alpha}_{0}<\boldsymbol{\alpha}
$$

Por tanto, por $\left(11^{\prime}\right)$,

$$
x_{0} \varepsilon V_{\alpha},
$$

en contradicción con (\#). Así, (14) está establecido.

Quisiera detenerme unos momentos para justificar la forma de emplear en esta prueba el esquema de sustitución para construir el conjunto A. Más formalmente, el argumento sería como sigue: Sea $\varphi(y, z)$ la fórmula que expresa:

$$
\begin{aligned}
& z \text { es un ordinal } y y \varepsilon \mathrm{V}_{z} \text { y para } \\
& \text { todo ordinal } w \varepsilon z, y \notin \mathrm{V}_{w} \text {. }
\end{aligned}
$$

Entonces

$$
A=\{z:(\exists y \varepsilon x)(\varphi(y, z))
$$

existe, por el axioma de sustitución.

\section{Conclusión}

Hemos regresado, pues, a nuestro punto de partida: la distribución de los conjuntos en una jerarquía bien ordenada de estratos. Cada conjunto aparece en un primer estrato $y$, desde ahí, continúa apareciendo 
en todos los estratos posteriores. $\mathbf{E l}$ ordinal correspondiente a su primera aparición puede ser tomado como una medida de complejidad del conjunto: conjuntos más simples aparecen en estratos anteriores; dos conjuntos que aparecen por primera vez en el mismo estrato poseerán la misma complejidad. El primer estrato, $V_{0}$, es vacio; el segundo estrato, $V_{1}$, contiene el conjunto vacio como único elemento, $V_{2}=\{\phi,\{\phi\}\}$, etc. Los estratos, finalmente, van sucediéndose indefinidamente en el dominio de lo transfinito, un estrato para cada ordinal, cofinalmente con el universo.

Puesto que dan lugar a esta estructurada jerarquia de los objetos de que tratan, los axiomas de Zermelo-Fraenkel no pueden considerarse simplemente una respuesta a las necesidades del momento, meros postulados que permiten "hacer matemática" al tiempo que esquivan las paradojas. Ésta es, tal vez, una conclusión a la que puede llegarse tras una inspección sumaria de sus axiomas (aunque el axioma de regularidad presenta problemas al respecto). Pero una excursión algo más profunda a través de sus consecuencias nos ha podido mostrar el gran contenido intuitivo de esta teoría.

Quedan, sin embargo, muchos problemas que resolver. Uno de ellos es la existencia de enunciados expresables en la teoria e indecidibles en ella. Sabemos por los teoremas de incompletud de Gödel que tales enunciados deben existir. Así, por ejemplo, el enunciado que, debidamente interpretado, afirma de sí mismo que no es un teorema, o el enunciado que expresa que la teoría es consistente. Sin embargo, sabemos (o creemos saber) que estos enunciados son verdaderos, ya que confiamos en la consistencia de la teoría (una razón para esta declaración de confianza es su neto carácter intuitivo).

Pero no es la existencia de enunciados indecidibles de este tipo lo que causa los mayores problemas. A fin de cuentas, los resultados de Gödel son una limitación necesaria con la que nos hemos habituado a coexistir. El problema real surge de la existencia de enunciados puramente conjuntistas indecidibles en la teoría. Si todo nuestro conocimiento matemático es reproducible en Zermelo-Fraenkel, entonces un enunciado independiente de esta teoría es, de hecho, un enunciado independiente de nuestro conocimiento. Creo que aquí se localiza un profundo problema en filosofía de la matemática.

\section{REFERENCIAS}

A. Levy, Basic Set Theory. Springer-Verlag, 1979.

D. Scotr, "Axiomatizing Set Theory", en American Mathematical Society Proceedings of Symposia in Pure Mathematics, vol. 13, part II (pp. 207-214). 